\title{
Pair production in differently polarized electric fields with frequency chirps
}

\author{
Obulkasim Olugh, ${ }^{1}$ Zi-Liang Li, ${ }^{2}$ Bai-Song Xie, ${ }^{1,3,{ }^{*}}$ and Reinhard Alkofer ${ }^{4, \dagger}$ \\ ${ }^{1}$ Key Laboratory of Beam Technology of the Ministry of Education, and College of Nuclear Science \\ and Technology, Beijing Normal University, Beijing 100875, China \\ ${ }^{2}$ School of Science, China University of Mining and Technology, Beijing 100083, China \\ ${ }^{3}$ Beijing Radiation Center, Beijing 100875, China \\ ${ }^{4}$ Institute of Physics, University of Graz, NAWI Graz, Universitätsplatz 5, 8010 Graz, Austria
}

(Received 30 November 2018; published 6 February 2019)

\begin{abstract}
Electron-positron pair production in strong electric fields, i.e., the Sauter-Schwinger effect, is studied using the real-time Dirac-Heisenberg-Wigner formalism. Hereby, the electric field is modeled to be a homogeneous, single-pulse field with subcritical peak field strength. Momentum spectra are calculated for four different polarizations - linear, elliptic, near-circular elliptic or circular-as well as a number of linear frequency chirps. With details depending on the chosen polarization, the frequency chirps lead to strong interference effects and thus quite substantial changes in the momentum spectra. The resulting produced pairs' number densities depend nonlinearly on the parameter characterizing the polarization and are very sensitive to variations of the chirp parameter. For some of the investigated frequency chirps, this can provide an enhancement of the number density by 3 to 4 orders of magnitude.
\end{abstract}

DOI: 10.1103/PhysRevD.99.036003

\section{INTRODUCTION}

Electron-positron $\left(e^{+} e^{-}\right)$pair production in strong electric fields, also known as the Sauter-Schwinger effect, is a long-standing theoretical prediction [1-3], which is, however, not yet experimentally verified; for a recent review, see, e.g., [4]. The pair production rate is hereby exponentially suppressed and proportional to $\exp \left(-\pi E_{c r} / E\right)$ as long as the electric field is of the order of or smaller than the critical field, $E_{c r}=m_{e}^{2} c^{3} / e \hbar \approx 1.3 \times 10^{18} \mathrm{~V} / \mathrm{m}$. The related laser intensity, e.g., $I=4.3 \times 10^{29} \mathrm{~W} / \mathrm{cm}^{2}$, is beyond current technological possibilities but the progress in highintensity laser technology [5-7] might make experimental tests possible in the next decade, especially in view of such planned facilities as the Extreme Light Infrastructure (ELI), the Exawatt Center for Extreme Light Studies (XCELS), and the Station of Extreme Light at the Shanghai Coherent Light Source. On the other hand, the already operating x-ray free electron laser (XFEL) at DESY in Hamburg can in principle achieve near-critical field strength as large as $E \approx 0.1 E_{c r}$; see, e.g., [8]. Triggered by the technical design report of the XFEL, numerical

\footnotetext{
*bsxie@bnu.edu.cn

reinhard.alkofer@uni-graz.at
}

Published by the American Physical Society under the terms of the Creative Commons Attribution 4.0 International license. Further distribution of this work must maintain attribution to the author(s) and the published article's title, journal citation, and DOI. Funded by SCOAP . estimates of the achievable number densities and of the resulting momentum spectra have been performed within the quantum kinetic approach at the beginning of the millenium [9,10], but many studies of the SauterSchwinger effect based on a number of different theoretical techniques have been undertaken in the last century and in this one; for a guide to the literature, we refer to the recent review [4].

Among the contributions from theorists towards an experimental verification of nonperturbative ultra-strongfield pair production, the dynamically assisted SauterSchwinger effect [11] deserves special mention. It exploits the idea that a combination of a low with a high frequency laser pulse leads to $e^{+} e^{-}$pair production rates which are by several orders of magnitude larger than the sum of the rates for the two separate pulses. Herein we report on a study which extends this idea by exploiting time-dependent frequencies, i.e., frequency chirps. We focus on linear chirps but allow then for different types of polarization. For simplicity, we study pair production in a single-pulse field with a Gaussian envelope:

$\mathbf{E}(t)=\frac{E_{0}}{\sqrt{1+\delta^{2}}} \exp \left(-\frac{t^{2}}{2 \tau^{2}}\right)\left(\begin{array}{c}\cos \left(b t^{2}+\omega t+\phi\right) \\ \delta \sin \left(b t^{2}+\omega t+\phi\right) \\ 0\end{array}\right)$,

where $E_{0} / \sqrt{1+\delta^{2}}$ is the amplitude of the electric field, $\tau$ denotes the pulse duration and $\omega$ the oscillation frequency at $t=0$. For completeness, we kept the carrier phase $\phi$ 
(which is known to have a significant effect on the momentum spectra of the produced pair $[12,13]$ ) in this expression, however, it will be set to zero in the following. Note that the above purely time-dependent electric fields (1) could be considered as the dipole approximation of the standing wave formed by two counterpropagating laser beams with different polarizations. The main interest in this study is the dependence on the chirp parameter $b$. Note that a nonvanishing $b$ can be interpreted as a time-dependent effective frequency, $\omega_{\text {eff }}=\omega+b t$. The effect of the chirp parameter $b$ on the time dependence of the electric field is displayed in Fig. 1. The parameter $\delta$ with $-1 \leq \delta \leq 1$ describes the ellipticity of the electric field, $\delta=0$ corresponds to linear and $\delta=1$ to circular polarization.

For the study presented here, the Dirac-HeisenbergWigner (DHW) formalism adapted to pair production $[14,15]$ is used. This choice is motivated due to its efficiency for calculations involving circularly or elliptically polarized electric background fields; see, e.g., [16,17], in which pair production in rotating circularly polarized electric fields has been investigated, or [18] for elliptically polarized fields. Without chirp the influence of the field ellipticity on pair production effects has been studied for various scenarios, we refer the interested reader to [26] for plane-wave fields and Ref. [27] for time-dependent electric fields, respectively.

At this point a remark with respect to the chosen polarizations for this study is in order. On the experimental side, due to limitations in instruments, it is much harder to produce a perfect circularly polarized field than an

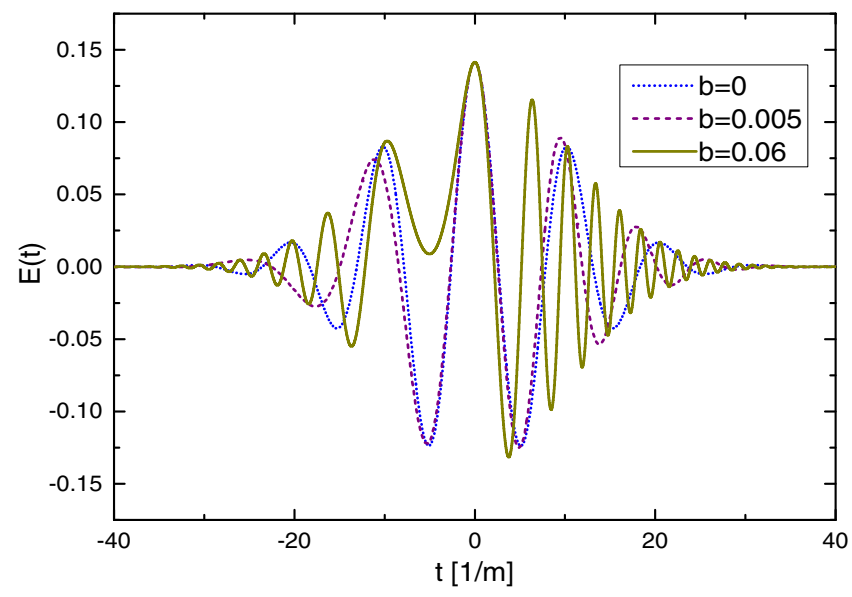

FIG. 1. The time dependence of the electric field $E(t)$ in units of the critical field for the linearly polarized $(\delta=0)$ case. The chosen parameters are $E_{0}=0.1 \sqrt{2} E_{c r}, \omega=0.6 \mathrm{~m}$, and $\tau=10 / m$ where $m$ is the electron mass. The blue dotted line shows the electric field without a chirp, $b=0$. The purple dashed line displays the field with a chirp parameter $b=0.005 \mathrm{~m}^{2}$, the dark yellow-green solid line for the chirp parameter $b=0.06 \mathrm{~m}^{2}$. These two values have been chosen for the chirp parameter because they present a typical "normal chirp" and a kind of maximally large chirp $b=\omega / \tau$, respectively. elliptically polarized and/or linearly polarized field. For low-intensity laser fields, a polarization of up to \pm 0.93 has been achieved experimentally [19]. Due to this, we include calculations for a near-circular elliptic polarization, i.e., for $\delta=0.9$.

In addition, we note that high-intensity laser pulses are also obtained through the chirped laser pulse amplification technique [20]. Therefore the study of pair production in fields with frequency chirps is well-motivated even besides the here found amplification similar to the one of the dynamically assisted Sauter-Schwinger effect.

Throughout this paper natural units $\hbar=c=1$ are used. Furthermore, three of the five parameters characterizing the electric field stay fixed:

$$
E_{0}=0.1 \sqrt{2} E_{c r} \quad \omega=0.6 m \quad \tau=10 / m,
$$

where $m$ is the electron mass. By introducing the Keldysh adiabaticity parameter $\gamma=m \omega /|e E|$, where $\omega$ and $E$ are the frequency and strength of the electric field, respectively, the Schwinger (tunneling) and the multi-photon pair production can be identified by $\gamma \ll 1$ and $\gamma \gg 1$, respectively [21]. Thus, for the given parameters in Eq. (2), the Keldysh parameter is $\gamma=4.25 \sqrt{1+\delta^{2}}$ for each polarization without chirp. For nonvanishing chirps, the effective Keldysh parameter at a given time is calculated by replacing $\omega$ with $\omega_{\text {eff }}(t)=\omega+b t$. For the chirp parameter $b$, we investigate several cases in the interval $0 \leq b \leq 0.06 \mathrm{~m}^{2}$, and for the polarization four different values of $\delta$ are chosen. We are aware that the pulse length is hardly sufficient to provide a clean multi-photon signal, and that a value of $b=0.06 \mathrm{~m}^{2}$ is already too large to be classified as a "normal chirp", however, the goal of the present exploratory study is a qualitative understanding of the influence of chirps on the produced number densities of pairs and the related momenta spectra for different polarizations, and to this end the chosen parameter sets are very suitable.

This paper is organized as follows: In Sec. II, we introduce briefly the DHW formalism to make the presentation reasonably self-contained. In Sec. III, we present our numerical results for the number densities for different chirp parameters and different polarizations. In Sec. IV, we summarize briefly for four polarizations how generic properties of the momentum spectra change for an increasing chirp parameter. In Sec. V, we reanalyze the spectra within a semiclassical treatment and discuss in how far the momentum spectra can be qualitatively understood. In the last section, we present our conclusions.

\section{THEORETICAL DESCRIPTION: THE DHW FORMALISM}

The here presented study employs the DHW formalism which is a relativistic phase-space approach. It has been further developed for the case of Sauter-Schwinger pair 
production in Refs. [14,15]. Within this method the electron is treated as a quantum field but the laser pulse is approximated by its mean-field which is justified by the magnitude of the used electric field.

To make this paper self-contained we briefly review the formalism. To this end we start from the gauge-invariant density operator of the system,

$$
\hat{\mathcal{C}}_{\alpha \beta}(r, s)=\mathcal{U}(A, r, s)\left[\bar{\psi}_{\beta}(r-s / 2), \psi_{\alpha}(r+s / 2)\right],
$$

in terms of the electron's spinor-valued Dirac field $\psi_{\alpha}(x)$, and $r$ denotes the center-of-mass and $s$ the relative coordinate. The Wilson line factor

$$
\mathcal{U}(A, r, s)=\exp \left(\mathrm{i} e s \int_{-1 / 2}^{1 / 2} d \xi A(r+\xi s)\right)
$$

renders the density operator gauge-invariant. Note that this factor depends on the elementary charge $e$ and the background gauge field $A$. The background field is treated in mean-field (Hartree) approximation, i.e.,

$$
F^{\mu \nu}(x) \approx\left\langle\hat{F}^{\mu \nu}(x)\right\rangle,
$$

and, because in a given Lorentz frame and gauge the background gauge field $A(\mathbf{x}, t)$ is a fixed $c$-number valued function, no path ordering is needed. The covariant Wigner operator,

$$
\hat{\mathcal{W}}_{\alpha \beta}(r, p)=\frac{1}{2} \int d^{4} s \mathrm{e}^{\mathrm{i} p s} \hat{\mathcal{C}}_{\alpha \beta}(r, s),
$$

thus includes the electron's quantum fluctuations but not the one of the electric field.

The simplification introduced by the mean-field approximation for the electromagnetic field becomes apparent when one considers the vacuum expectation value of the covariant Wigner operator to obtain the covariant Wigner function

$$
\mathbb{W}(r, p)=\langle\Phi|\hat{\mathcal{W}}(r, p)| \Phi\rangle
$$

In the equation of motion of this correlation function, the electromagnetic field factors out:

$$
\left\langle\Phi\left|F_{\mu \nu} \hat{\mathcal{C}}\right| \Phi\right\rangle=F_{\mu \nu}\langle\Phi|\hat{\mathcal{C}}| \Phi\rangle .
$$

This in turn allows to terminate the in general infinite hierarchy of correlation functions.

As the Wigner function is a Dirac-matrix valued quantity it can be decomposed into 16 covariant Wigner coefficients

$$
\mathbb{W}=\frac{1}{4}\left(\mathbb{1} \mathbb{S}+\mathrm{i} \gamma_{5} \mathbb{P}+\gamma^{\mu} \mathbb{V}_{\mu}+\gamma^{\mu} \gamma_{5} \mathbb{A}_{\mu}+\sigma^{\mu \nu} \mathbb{T}_{\mu \nu}\right)
$$

Hereby, the related spin and parity properties are made evident by the notation. As the modeling of the electric field already indicates we work in a definite frame. Correspondingly, one can project on equal times which yields the equal-time Wigner function

$$
\mathbb{W}(\mathbf{x}, \mathbf{p}, t)=\int \frac{d p_{0}}{2 \pi} \mathbb{W}(r, p)
$$

and by an analogous decomposition to Eq. (9) the corresponding equal-time Wigner coefficients $\mathbb{s}, \mathbb{P}, \mathbb{v}_{0, x, y, z}$ etc.

As the equations of motions for the Wigner coeffecients are quite lengthy we refrain from repeating the respective formula here. Their explicit form as well as detailed derivations can be found in [15,22]. A decisive advantage of employing Wigner coefficients is given by the relation of $\mathbb{s}$ with the mass, of $\mathbb{v}_{0}$ with the charge, and of $\vec{v}$ as current density in the case without electric field $[14,15]$. Correspondingly, one chooses vacuum initial conditions as starting values. The nonvanishing values are

$$
\mathbb{S}_{\mathrm{vac}}=\frac{-2 m}{\sqrt{\mathbf{p}^{2}+m^{2}}}, \quad \mathbb{v}_{i, \mathrm{vac}}=\frac{-2 p_{i}}{\sqrt{\mathbf{p}^{2}+m^{2}}} .
$$

In general, the equations of motions for the Wigner coefficients are integro-differential equations. Their numerical solution is due to the challenging nonlocal nature of the respective pseudodifferential operators; see, e.g., [15, 22-24]. For the homogeneous electric field (1) studied here, these equations can be reduced to ordinary differential equations [16]. To this end, we note first that, at most, ten out of the sixteen Wigner coefficients are nonvanishing:

$$
\mathbb{W}=\left(\mathbb{s}, \mathbb{v}_{i}, \circledast_{i}, \mathbb{t}_{i}\right), \quad \mathbb{t}_{i}:=\mathbb{t}_{0 i}-\mathbb{t}_{i 0} .
$$

Second, the kinetic momentum $\mathbf{p}$ is related to the canonical momentum $\mathbf{q}$ via

$$
\mathbf{p}(t)=\mathbf{q}-e \mathbf{A}(t)
$$

and is, thus, time dependent. In a next step, one expresses the scalar Wigner coefficient by the one-particle distribution function $f(\mathbf{q}, t)$. The latter is related to the phase-space energy density,

$$
\varepsilon=m s+p_{i} \mathbb{v}_{i}
$$

via

$$
f(\mathbf{q}, t)=\frac{1}{2 \Omega(\mathbf{q}, t)}\left(\varepsilon-\varepsilon_{\mathrm{vac}}\right) .
$$

Hereby, $\Omega(\mathbf{q}, t)=\sqrt{\mathbf{p}^{2}(t)+m^{2}}=\sqrt{m^{2}+(\mathbf{q}-e \mathbf{A}(t))^{2}}$ is the electron's (positron's) energy.

In addition, it is helpful to define an auxiliary threedimensional vector $\mathbf{v}(\mathbf{q}, t)$ : 
$v_{i}(\mathbf{q}, t):=\mathbb{v}_{i}(\mathbf{p}(t), t)-(1-f(\mathbf{q}, t)) \mathbb{v}_{i, \text { vac }}(\mathbf{p}(t), t)$.

The one-particle momentum distribution function $f(\mathbf{q}, t)$ can then be obtained by solving the following ten ordinary differential equations for $f(\mathbf{q}, t)$ and the nine auxiliary quantities $v_{i}(\mathbf{q}, t), a_{i}(\mathbf{q}, t):=\mathbb{\boxplus}_{i}(\mathbf{q}, t)$ and $t_{i}(\mathbf{q}, t):=\mathbb{t}_{i}(\mathbf{q}, t)$ :

$\dot{f}=\frac{e}{2 \Omega} \mathbf{E} \cdot \mathbf{v}$,

$\dot{\mathbf{v}}=\frac{2}{\Omega^{3}}\left((e \mathbf{E} \cdot \mathbf{p}) \mathbf{p}-e \Omega^{2} \mathbf{E}\right)(f-1)-\frac{(e \mathbf{E} \cdot \mathbf{v}) \mathbf{p}}{\Omega^{2}}$

$-2 \mathbf{p} \times \mathbf{a}-2 m \mathbf{t}, \dot{\mathbf{a}}=-2 \mathbf{p} \times \mathbf{v}, \dot{\mathbf{t}}=\frac{2}{m}\left[m^{2} \mathbf{v}-(\mathbf{p} \cdot \mathbf{v}) \mathbf{p}\right]$,

where as usual the dot is a shorthand for the time derivative. Together with the initial conditions $f(\mathbf{q},-\infty)=0$, $\mathbf{v}(\mathbf{q},-\infty)=\mathbf{a}(\mathbf{q},-\infty)=\mathbf{t}(\mathbf{q},-\infty)=0$, this set of
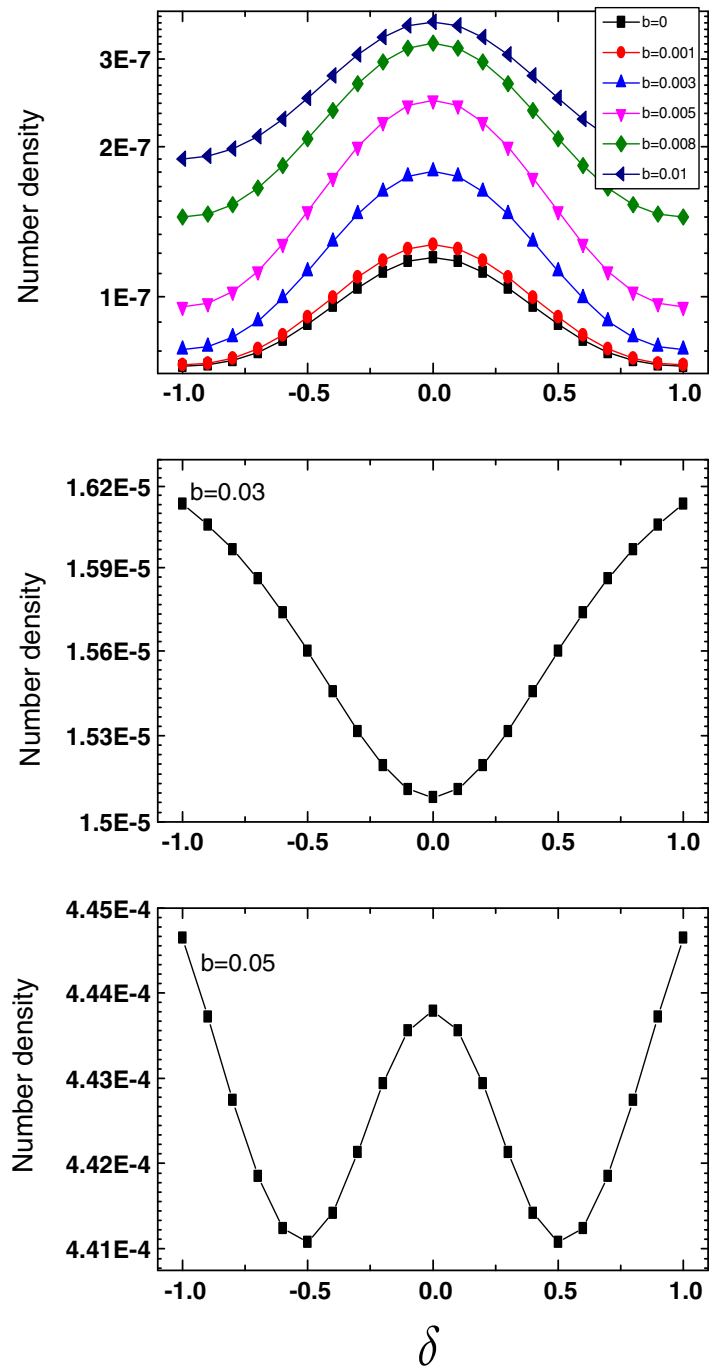

equations is a well-defined and numerically straightforward solvable initial value problem.

The number density of created pairs is obtained by integrating the distribution function $f(\mathbf{q}, t)$ over all momenta at asymptotically late times $t \rightarrow+\infty$ :

$$
n=\lim _{t \rightarrow+\infty} \int \frac{d^{3} q}{(2 \pi)^{3}} f(\mathbf{q}, t) .
$$

\section{NUMERICAL RESULTS FOR NUMBER DENSITIES}

As already stated the carrier phase is chosen to be $\phi=0$ leaving studies similar to the one presented here but with nonvanishing carrier phase for future investigations. Herein, we examine the main results for the number density of the produced particles for several chirp parameters for the different polarization.
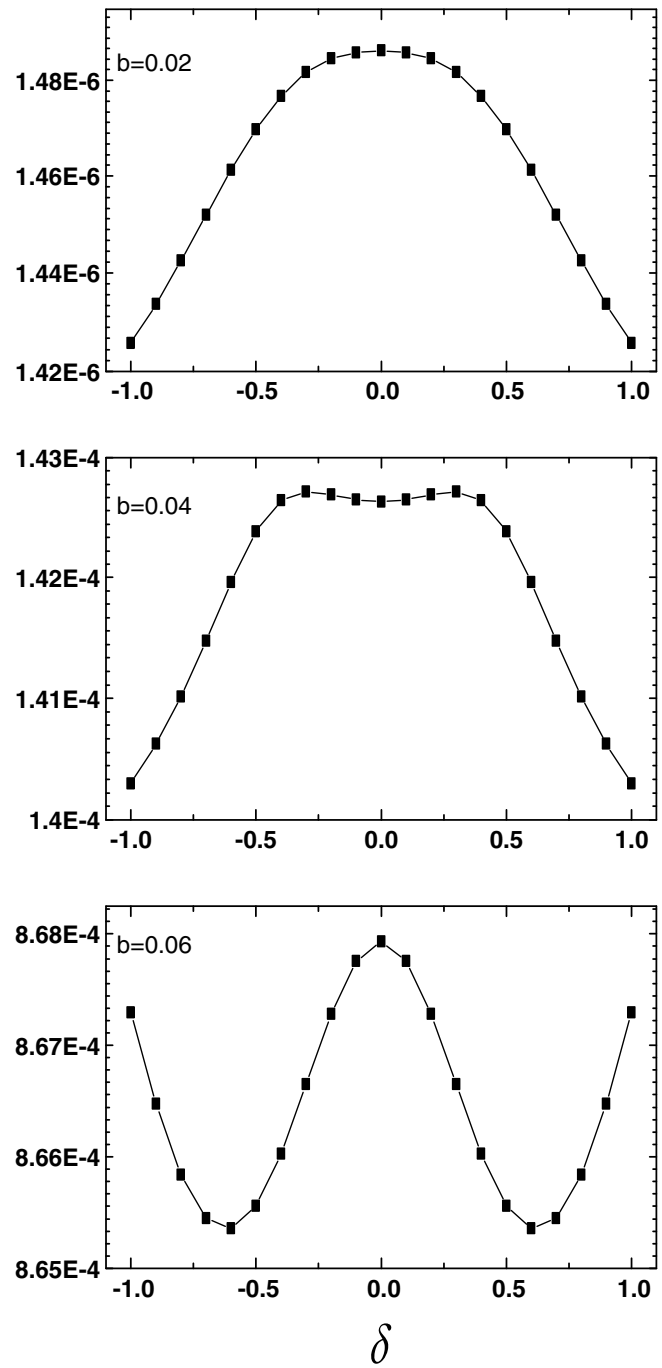

FIG. 2. The number density (in units of $\lambda_{c}^{-3}=m^{3}$ ) of created particles as a function of the field polarization $\delta$ for different chirp parameters $b$. The other field parameters are the same as in Fig. 1. 
The number densities as a function of the polarization parameter $\delta$ are shown in Fig. 2. They clearly display the expected symmetry when mirroring $\delta \rightarrow-\delta$. This then implies that the case of linear polarization, $\delta=0$, provides an extremum in the number density. As one immediately sees from Fig. 2, the maximum which is present at small chirps becomes a minimum at larger values of $b$ which then turns into a maximum again for even larger $b$-values. Additional extrema appear for very large values, $b \geq 0.04$ $\mathrm{m}^{2}$. However, the more important effects are the following two: First, with increasing chirp the relative variation in the number density becomes much smaller. For vanishing chirp, the ratio of the number density for linear polarization to the one for circular polarization is more than a factor of two. At $b=0.06 \mathrm{~m}^{2}$ the largest number density deviates from the smallest one (assumed at $\delta \approx \pm 0.6$ ) by less than three per mille. Second, with increasing chirp the peak number density increases significantly. This effect is most pronounced when increasing from $b=0.02 \mathrm{~m}^{2}$ to $b=$ $0.03 \mathrm{~m}^{2}$ for which the number density increases by more than a factor of ten for all polarizations.

When plotting the number densities as a function of the chirp parameter $b$ for the three different polarizations $\delta=0,0.5$ and 1 , one sees for relatively small chirp values a symmetry under the reflection $b \rightarrow-b$; see the upper panel of Fig. 3. Also very clearly visible is the suppression in the number densities when one goes from linear to circular polarization. From the lower panel of Fig. 3, one can infer the more or less exponential increase in number density for increasing chirp as well as the fact that the number densities for different polarization become degenerate for different polarizations. As the effective frequency $\omega_{\text {eff }}=\omega+b t$ increases towards the end of the pulse, see Fig. 1, a related increase in the production rate is expected. Nevertheless, the size of the effect is surprisingly large. The physical reason is that if a frequency chirp, as the one in Eq. (1), is applied, the electric field contains components of significantly higher frequencies as compared to the frequency $\omega$ for the employed values of the chirp parameter $b$. From the definition of the Keldysh adiabaticity parameter $\gamma=\frac{m \omega}{|e E|}$, one can see that for a fixed field strength $E$ it only depends on the field's frequency. As for the applied frequency chirp, the field's effective frequency increases with time the effective Keldysh parameter also increases within the time of the pulse duration, and for most of the chosen parameters it will thereby change from values smaller than one to values much larger than one. As the multiphoton pair production rate is substantially larger than the Sauter-Schwinger one, the addition of a chirp leads to the dominance of the higher frequency components of the pulse in pair creation, and this is valid for each polarization. Therefore, the resulting particle creation is the dominated by "late-time" multiphoton processes due to the field's high-frequency components, and in the chirped electric field the production rate is enhanced significantly due to the
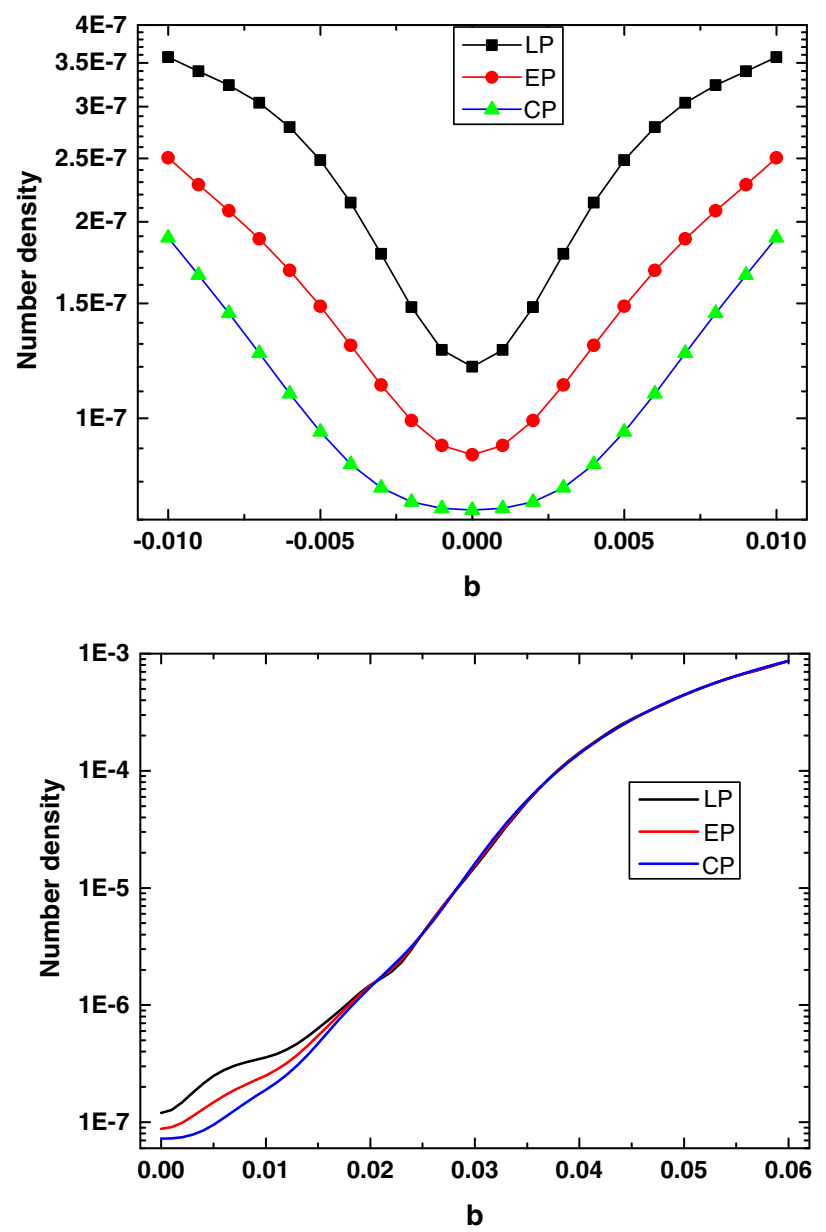

FIG. 3. The number density (in units of $\lambda_{c}^{-3}=m^{3}$ ) of created particles as a function of the chirp parameters $b$ for different polarizations $\delta=0(\mathrm{LP}), \delta=0.5(\mathrm{EP})$, and $\delta=1(C P)$, respectively. The other field parameters are the same as in Fig. 1.

increase of the effective frequency. Some corresponding numbers are provided in Table I

Here, a technical remark is in order. For some large values of the chirp parameter $b$, e.g., around $b=0.05 \mathrm{~m}^{2}$, some irregular changes of the numerical results for the number densities for each polarization are observed. As these have to be very likely attributed to instabilities of the numerical procedure we are refraining from displaying these exceptional points here. Nevertheless, this issue will be clarified in future investigations.

TABLE I. Numerical results for the number densities (in units of $\lambda_{c}^{-3}=m^{3}$ ) for some selected chirp (in units of $\mathrm{m}^{2}$ ) and polarization parameters.

\begin{tabular}{lcccc}
\hline \hline$n$ & $b=0$ & $b=0.02$ & $b=0.04$ & $b=0.06$ \\
\hline$\delta=0$ & $1.200 \times 10^{-7}$ & $1.486 \times 10^{-6}$ & $1.426 \times 10^{-4}$ & $0.8679 \times 10^{-3}$ \\
$\delta=0.5$ & $0.880 \times 10^{-7}$ & $1.470 \times 10^{-6}$ & $1.424 \times 10^{-4}$ & $0.8656 \times 10^{-3}$ \\
$\delta=0.9$ & $0.728 \times 10^{-7}$ & $1.434 \times 10^{-6}$ & $1.406 \times 10^{-4}$ & $0.8665 \times 10^{-3}$ \\
$\delta=1$ & $0.724 \times 10^{-7}$ & $1.426 \times 10^{-6}$ & $1.403 \times 10^{-4}$ & $0.8673 \times 10^{-3}$ \\
\hline \hline
\end{tabular}




\section{MOMENTUM SPECTRA}

In this section, we will report on results for the momenta spectra (MS) of the produced particles for several chirp parameters each for the cases of (i) linear polarization $(\delta=0)$, (ii) elliptical polarization $(\delta=0.5)$, (iii) nearcircular elliptical polarization $(\delta=0.9)$, and (iv) circular polarization $(\delta=1)$

\section{A. Momentum spectra for linear polarization $\delta=0$}

First of all, we note that in the case of linear polarization the electric field is oriented only along the $x$ axis, and the momentum spectra possess correspondingly a rotational symmetry around the $q_{x}$ axis. For the linear polarized $(\delta=0)$ pulse, the momentum spectra in $q_{x}$ and $q_{y}$ for $q_{z}=0$ are plotted in Fig. 4. For vanishing chirp, $b=0$, the results agree with the ones of a previous investigation [25]. For nonvanishing chirp parameters, the main result is, besides the expected lower symmetry of the spectra, the appearance of strong interference effects leading to several maxima and minima of the pair production rate as a function of momenta.

As can be seen in the upper panel of Fig. 4, small chirp parameters lead to small variations in the spectrum: There is a slight enhancement in the height of the peak, a small shift towards positive $q_{x}$, and a broadening as well as the loss of one of the reflection symmetries of the peak.
For larger frequency chirps, $b \geq 0.02 \mathrm{~m}^{2}$, the momentum spectra display some remarkable structures as can be seen from the lower row in Fig. 4. For $b=0.02 \mathrm{~m}^{2}$, the spectrum possesses two peaks at negative and positive $q_{x}$, respectively. For $b=0.03 \mathrm{~m}^{2}$, the main peak is located at negative $q_{x}$. For $b=0.04 m^{2}$, the peak goes back to positive momenta, and at $b=0.06 \mathrm{~m}^{2}$ again to negative momenta. In the latter case, strong interference effects are visible; note especially the ringlike structure. These quantum interferences in between the amplitudes describing pair creation relate to different cycles in the electric field. As pair creation is a non-Markovian process the accumulated phases depend on the complete earlier history, and therefore small changes in the field parameters in general change the relative phases of the amplitudes significantly. We will return to this point within the context of a semiclassical analysis in the discussion of Fig. 10 in Sec. V.

In all the cases shown in Fig. 4, the spectra are symmetric with respect to reflection of $q_{y}$. (NB: For nonvanishing $q_{z}$ the spectra would be symmetric with respect to a rotation around the $q_{x}$ axis.) Therefore, the effects of chirps can be understood in more detail by plotting the number density for $q_{y}=q_{z}=0$ as a function of $q_{x}$. In the left panel of Fig. 5(a), one sees that for vanishing chirp the peak is located at $q_{x}=0$. As $b$ increases, the peak is very slightly shifted to positive $q_{x}$. For $b=0.02 \mathrm{~m}^{2}$, one sees now not only the two prominent maxima but also additional but less
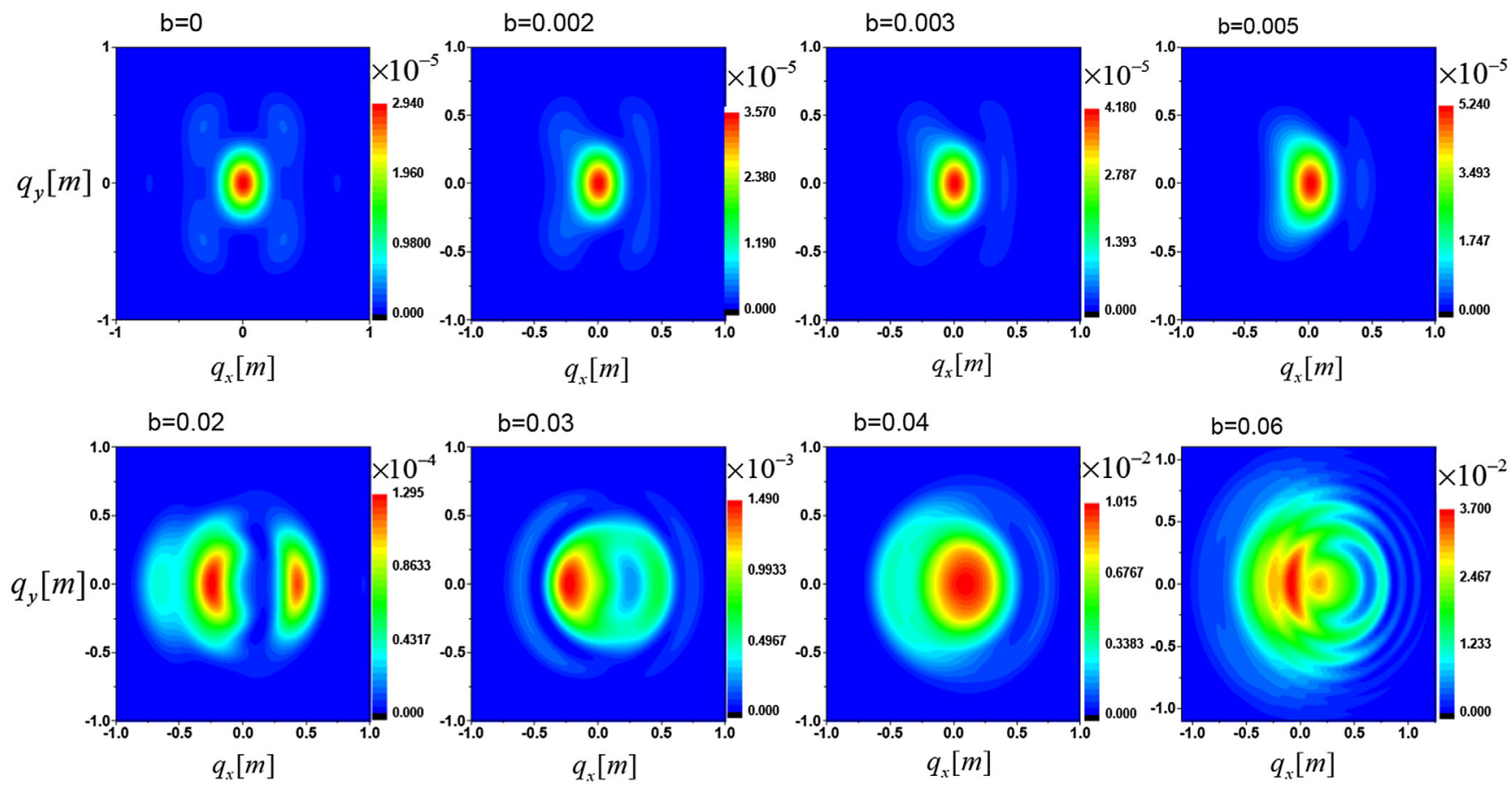

FIG. 4. Momentum spectra of produced $e^{+} e^{-}$pairs for linear polarization $(\delta=0)$ at $q_{z}=0$ in the $\left(q_{x}, q_{y}\right)$-plane. The other field parameters are given in Eq. (2). Upper row: from left to right the values of the small chirp parameters are $b=0,0.002 \mathrm{~m}^{2}, 0.003 \mathrm{~m}^{2}$ and $0.005 \mathrm{~m}^{2}$, respectively. Lower row: from left to right the values of the large chirp parameters are $b=0.02 \mathrm{~m}^{2}, 0.03 \mathrm{~m}^{2}, 0.04 \mathrm{~m}^{2}$ and $0.06 m^{2}$, respectively. 

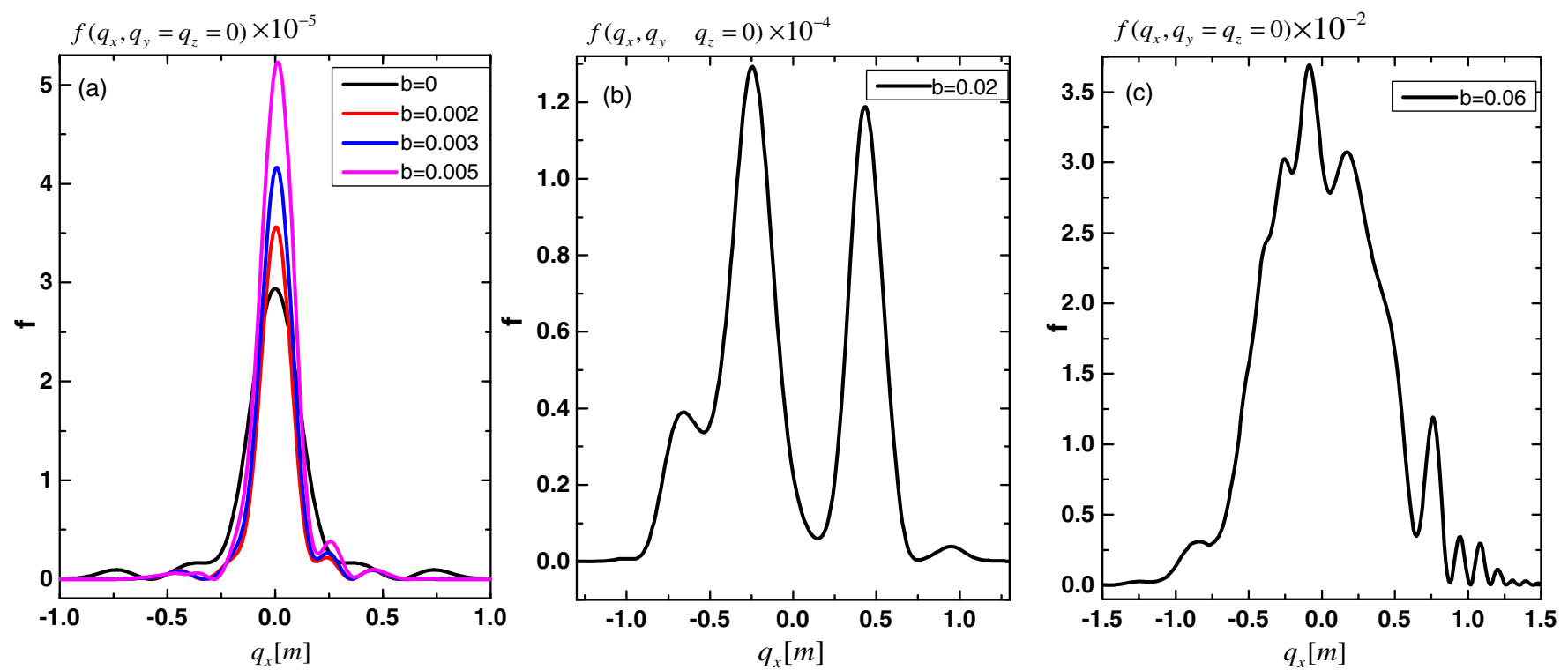

FIG. 5. Momentum spectra of produced pairs for linear polarization $(\delta=0)$ at $q_{y}=q_{z}=0$, cf. Fig. 4 .

pronounced ones. At $b=0.06 \mathrm{~m}^{2}$ the momentum spectrum displays quite complicated interference patterns but note also the change in the height of the peaks, enhanced from $2.94 \times 10^{-5}(b=0)$ to $3.7 \times 10^{-2}\left(b=0.06 m^{2}\right)$.

As we will argue in Sec. $V$ the effects of the frequency chirp on the spectra can be explained by a semiclassical analysis based on the WKB approximation. As the related effective potential changes with the frequency chirp the numerically observed drastic effects are plausible.

\section{B. Elliptical polarization $\delta=0.5$}

As a next case, we consider the momentum spectra for an elliptically polarized electric field, $\delta=0.5$; see Fig. 6. For
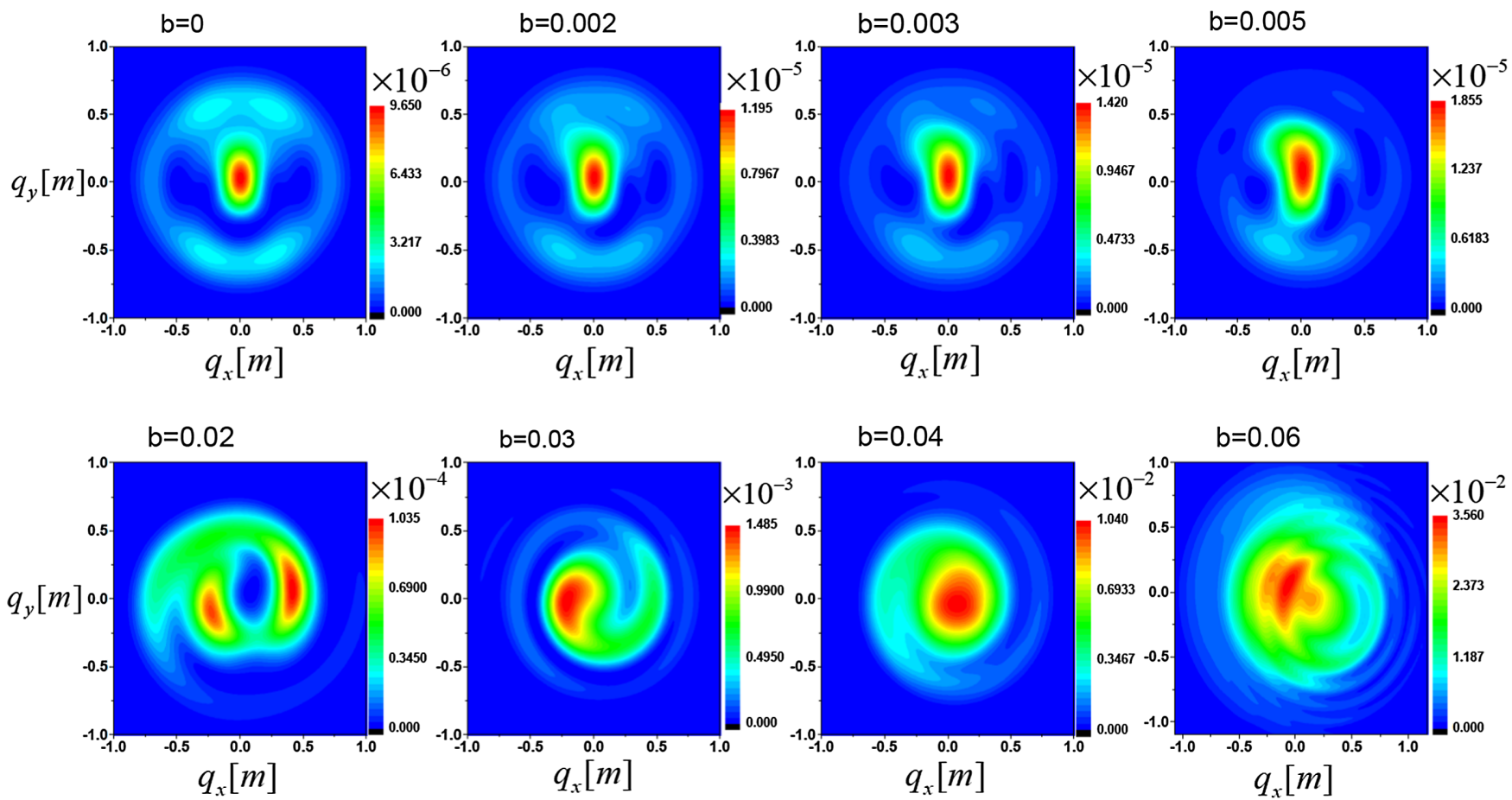

FIG. 6. Momentum spectra of produced $e^{+} e^{-}$pairs for elliptic polarization $(\delta=0.5)$ at $q_{z}=0$ in the $\left(q_{x}, q_{y}\right)$-plane. The other field parameters are given in Eq. (2). Upper row: from left to right the values of the chirp parameters are $b=0,0.002 \mathrm{~m}^{2}, 0.003 \mathrm{~m}^{2}$ and $0.005 \mathrm{~m}^{2}$, respectively. Lower row: from left to right the values of the chirp parameters are $b=0.02 \mathrm{~m}^{2}, 0.03 \mathrm{~m}^{2}, 0.04 \mathrm{~m}^{2}$ and $0.06 \mathrm{~m}^{2}$, respectively. 

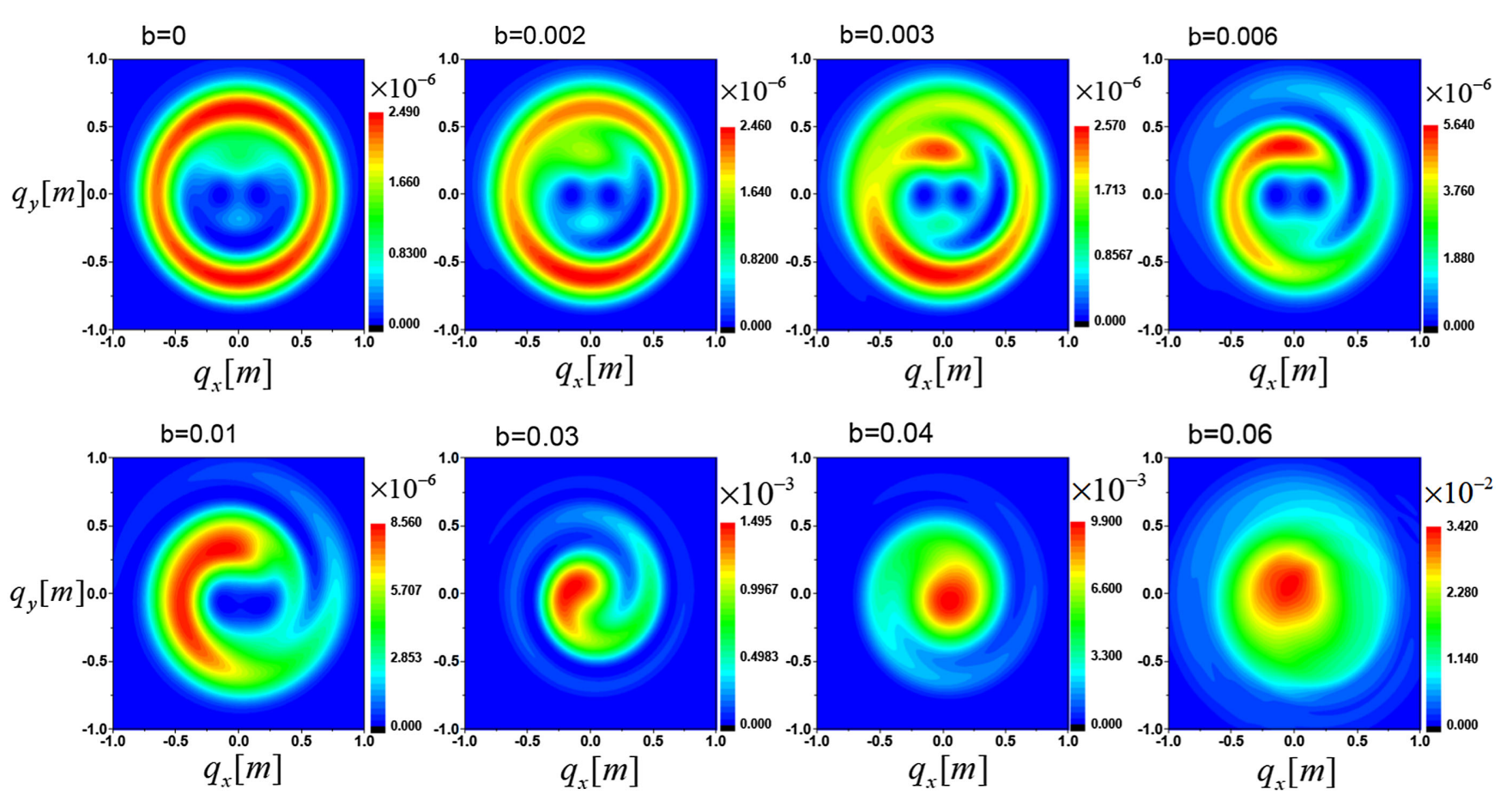

FIG. 7. Momentum spectra of produced $e^{+} e^{-}$pairs for near-circular elliptic polarization $(\delta=0.9)$ at $q_{z}=0$ in the $\left(q_{x}, q_{y}\right)$-plane. The other field parameters are given in Eq. (2). Upper row: from left to right the values of the chirp parameters are $b=0,0.002 m^{2}, 0.003 m^{2}$ and $0.006 \mathrm{~m}^{2}$, respectively. Lower row: from left to right the values of the chirp parameters are $b=0.01 \mathrm{~m}^{2}, 0.02 \mathrm{~m}^{2}, 0.03 \mathrm{~m}^{2}$ and $0.06 \mathrm{~m}^{2}$, respectively.
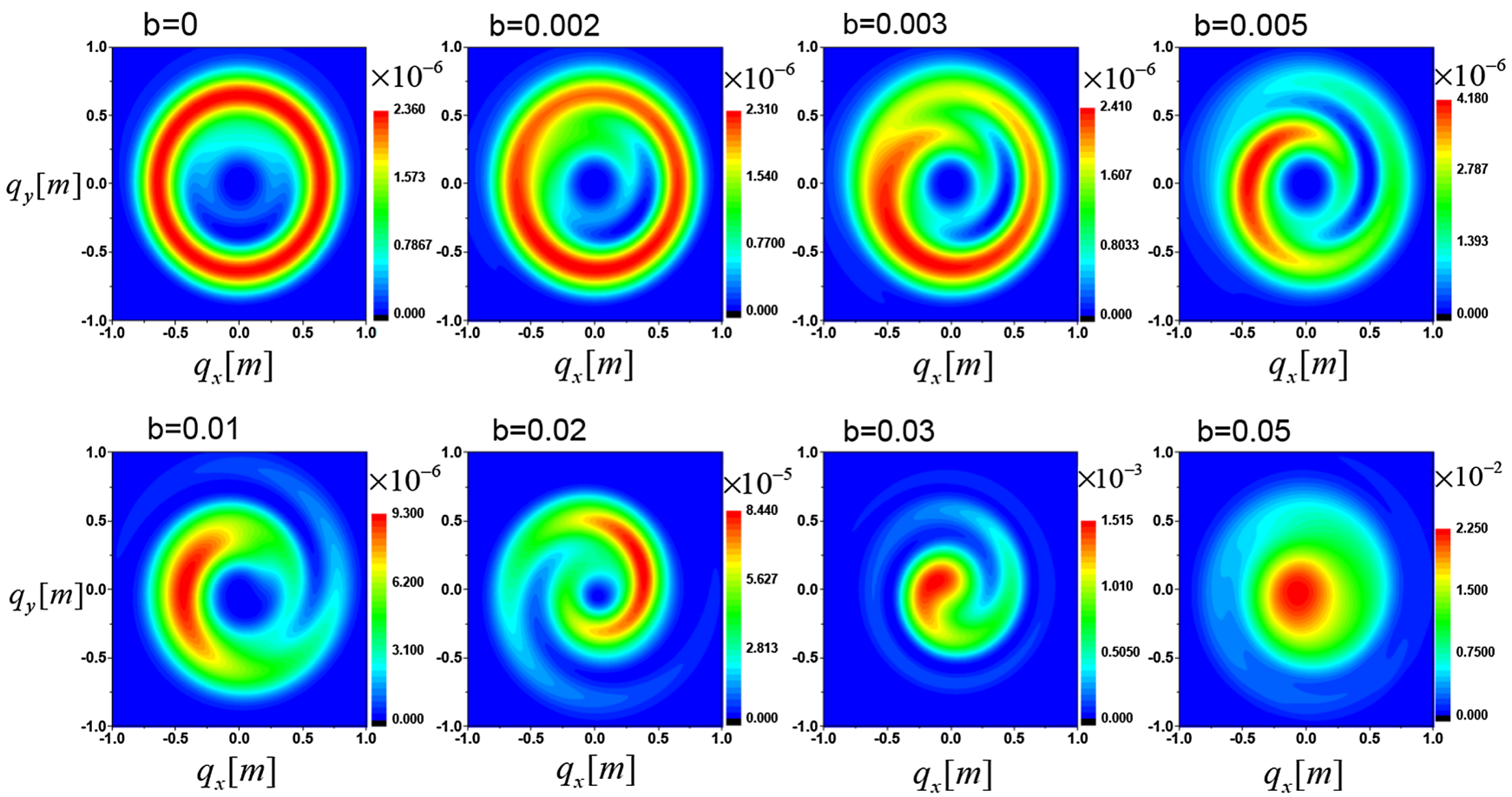

FIG. 8. Momentum spectra of produced $e^{+} e^{-}$pairs for circular polarization $(\delta=1)$ at $q_{z}=0$ in the $\left(q_{x}, q_{y}\right)$-plane. The other field parameters are given in Eq. (2). Upper row: from left to right the values of the chirp parameters are $b=0,0.002 \mathrm{~m}^{2}, 0.003 \mathrm{~m}^{2}$ and $0.005 \mathrm{~m}^{2}$, respectively. Lower row: from left to right the values of the chirp parameters are $b=0.01 \mathrm{~m}^{2}, 0.02 \mathrm{~m}^{2}, 0.03 \mathrm{~m}^{2}$ and $0.05 \mathrm{~m}^{2}$, respectively. 
$b=0$, however, there is still a reflection symmetry around the $q_{x}$ axis, and this symmetry gets lost when $b \neq 0$. For small chirp parameters, the distortion of the spectrum is again quite mild; see the upper panel of Fig. 6. Quite some complicated reordering of the spectra take place for large values of the chirp parameter, cf., the lower panel of Fig. 6. In particular, the splitting into several extrema is very similar to what happens in the linear polarized case.

\section{Near-circular elliptic polarization $\delta=0.9$}

For the near-circular elliptically polarized case we plot the spectra in Fig. 7. For $b=0$ the main peak region is ring-shaped. This can be understood from the fact that the electric field changes its direction during the pair creation process. Thus, the particles may be accelerated into different directions depending on the field direction at the time of
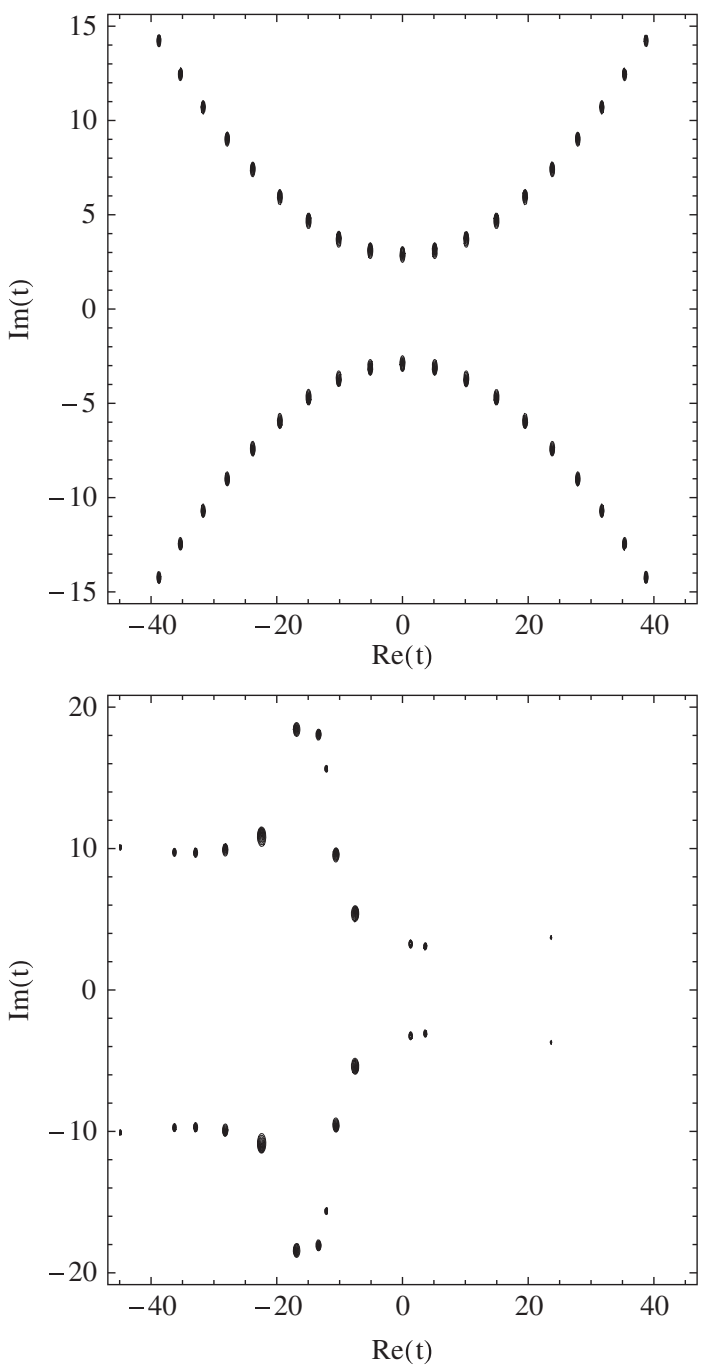

TABLE II. The peak values for the one-particle distribution function at late times $f(\mathbf{q}, \infty)$. Note that these peaks occur at different values of the momentum $\mathbf{q}$; see the discussion above.

\begin{tabular}{lcc}
\hline \hline$f(\mathbf{q}, \infty)$ at peak & $b=0$ & $b=0.06 \mathrm{~m}^{2}$ \\
\hline$\delta=0$ & $29.4 \times 10^{-6}$ & $3.70 \times 10^{-2}$ \\
$\delta=0.5$ & $9.65 \times 10^{-6}$ & $3.56 \times 10^{-2}$ \\
$\delta=0.9$ & $2.49 \times 10^{-6}$ & $3.42 \times 10^{-2}$ \\
$\delta=1$ & $2.36 \times 10^{-6}$ & $3.41 \times 10^{-2}$ \\
\hline \hline
\end{tabular}

production. These findings are very similar to the results of the strong-field ionization of helium using an elliptically polarized laser pulses [28] and the effects of electric field polarizations on pair production from vacuum [18].

Otherwise, one sees also drastic effects of the chirp for relatively small chirp parameters, and especially the ring
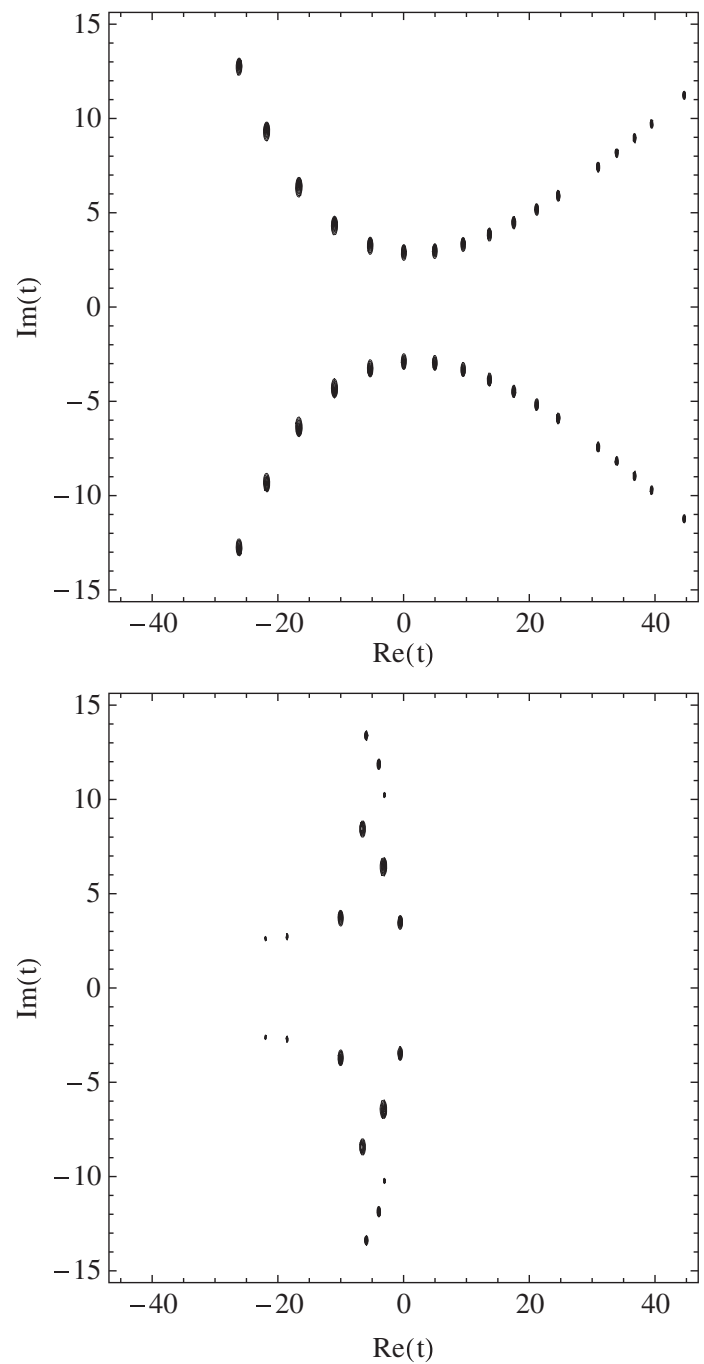

FIG. 9. Contour plots of $|\Omega(\mathbf{q}, t)|^{2}$ in the complex $t$ plane, showing the location of turning points where $\Omega(\mathbf{q}, t)=0$. These plots are for the linear polarization $\delta=0$. The other field parameters are $E_{0}=0.1 \sqrt{2} E_{c r}, \omega=0.6 m$, and $\tau=10 / m$. From top left to bottom right the values of chirp parameters are $b=0,0.005,0.02,0.06 \mathrm{~m}^{2}$, respectively, and momentum values are $q_{x}=0,0,-0.25,-0.11 m$, respectively. 
form is distorted to a spiral one. For very large chirp parameters, on the other hand, the spectrum shows in this case less structure.

In contrast to the previously discussed linear polarization in the case of the near-circular elliptic polarization, the spectra lose their symmetry in both of $q_{x}$ and $q_{y}$. The patterns observed in Fig. 7 will become clearer when discussing the case of perfect circular polarization.

\section{Circular polarization $\delta=1$}

For the circularly polarized $(\delta=1)$ the spectra are shown in Fig. 8. The $b=0$ spectrum, shown at the top and most left, displays a ring-shaped maximum centered around the origin. It exhibits the weak interference pattern or/and oscillation between the hole and outer ring along negative values of $q_{y}[16,18,25]$. This might be interpreted within a
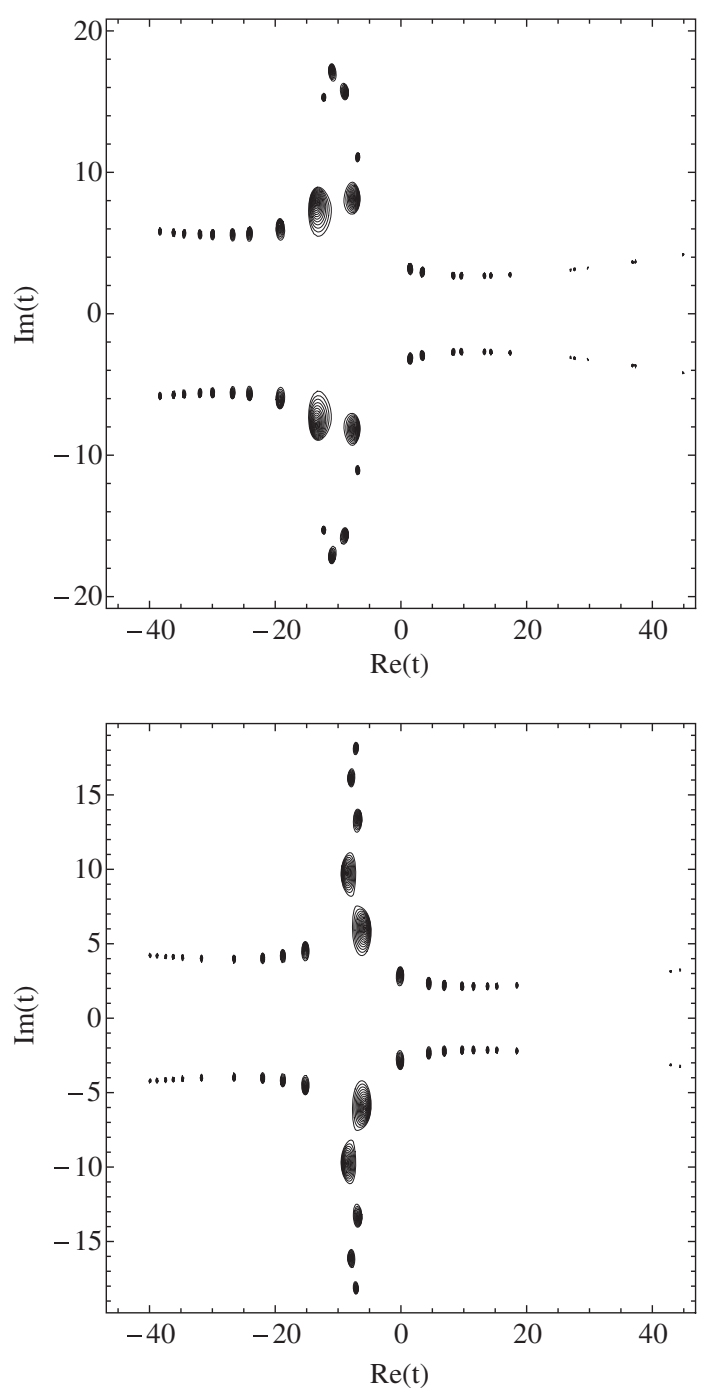

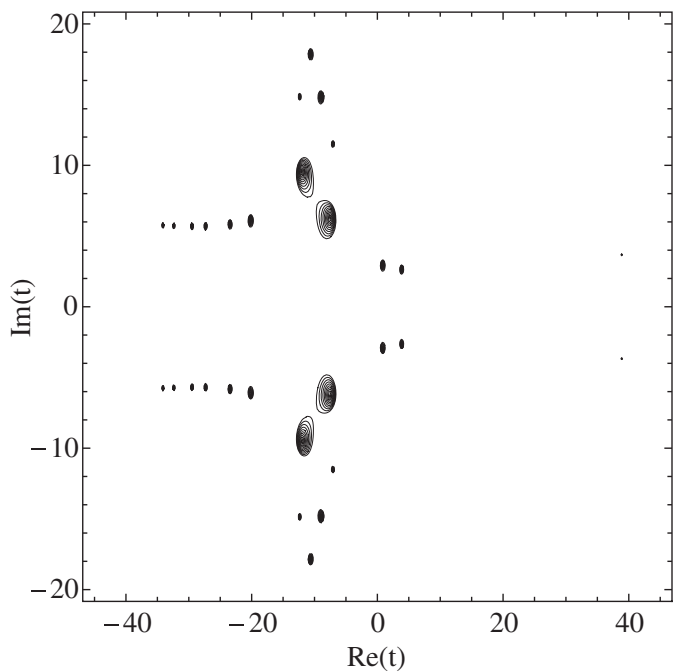

semiclassical analysis by means of an effective scattering potential [29]; see the next section. The outer ring structure results from multiphoton pair creation by absorbing four $(n=4)$ photons [16], and in the strong field limit its radius can be determined by the energy conservation to be at $|\mathbf{q}|=$ $1 / 2 \sqrt{(n \omega)^{2}-4 m^{2}}$ where $n$ is the number of photons participating in the pair creation, cf. Ref. [30].

Again the spectra are very sensitive to chirps even for relatively small chirp parameters. This includes the distortion of the ring structure, the appearance of spirals, and, last but not least, a significant increase of the one-particle distribution function. The characteristic shape of the spectra could be helpful in an experimental identification of pair production. For example, at $b=0.03 \mathrm{~m}^{2}$, one clearly identifies an Archimedian spiral which is going to start from almost the central region slightly shifted to negative

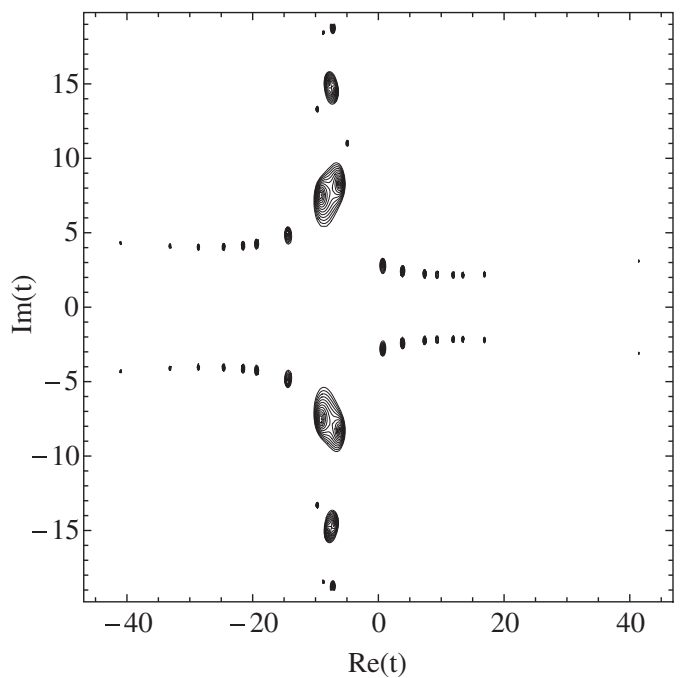

FIG. 10. Contour plots of $|\Omega(\mathbf{q}, t)|^{2}$ in the complex $t$ plane, showing the location of turning points where $\Omega(\mathbf{q}, t)=0$. These plots are for the linear polarization $\delta=0$. The other field parameters are $E_{0}=0.1 \sqrt{2} E_{c r}, \omega=0.6 m$, and $\tau=10 / m$. From top left to bottom right the values of chirp parameters are $b=0.03,0.03,0.04,0.04 \mathrm{~m}^{2}$, respectively, and momentum values are $q_{x}=-0.24,0.32,0.13,-0.4$ $m$, respectively. 
$q_{x}$ values. For very large chirp parameters, $b \geq 0.05 \mathrm{~m}^{2}$, the spiral structure is fading away, and the spectra become less structured.

To summarize this section, we have obtained quite some detailed information how the spectra of the produced pairs change for a given polarization when frequency chirps from relatively modest to quite large ones are considered. The positions of the global extrema of the one-particle distribution function, i.e., the peaks of the spectra display a quite rich structure. Common to all the considered cases is the strong increase in peak values for increasing chirp parameters which is easily understood from the effective frequency, $\omega_{\text {eff }}=\omega+b t$, of the field, respectively, by the onset of multi-photon pair production. Contrary to the case of a vanishing chirp parameter and thus of a constant frequency $\omega=0.6 \mathrm{~m}$ a sizable chirp parameter implies that the electric field contains higher frequency components.
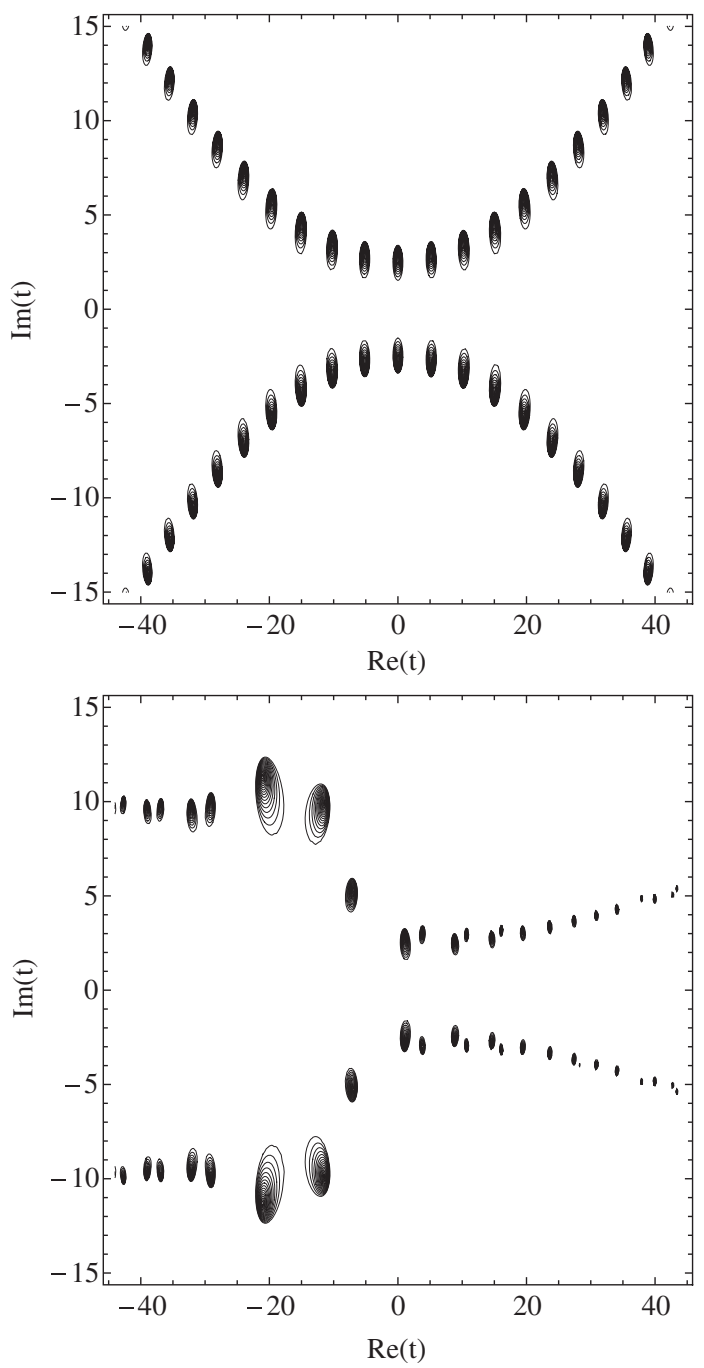

The "late-time" field resembles then an almost clean multiphoton signal even for the considered short pulse duration, and thus pair creation is dominated by multi-photon mechanism. Some corresponding values are given in Table II.

\section{SEMICLASSICAL ANALYSIS}

In this section, we will employ a semiclassical analysis to obtain a qualitative explanation of the effects of chirps on the spectrum. Hereby, we follow the WKB method outlined in Ref. [31]. These authors considered a Sauter pulse, i.e., a gauge potential $A(t) \propto \tanh \omega t$, for which the turning points of the analogue semiclassical scattering potential can be determined analytically by solving the condition $\Omega\left(\mathbf{q}, t_{p}\right)=\sqrt{m^{2}+\left(\mathbf{q}-e \mathbf{A}\left(t_{p}\right)\right)^{2}}=0$. In this case, a
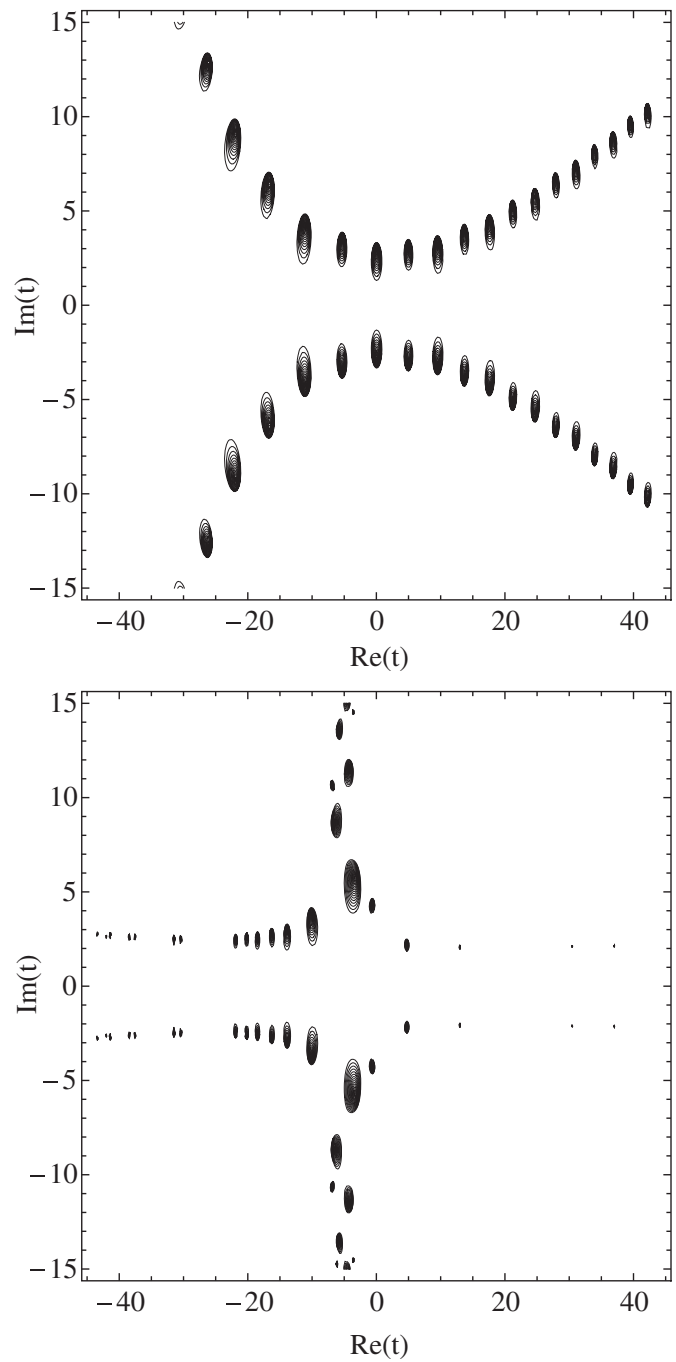

FIG. 11. Contour plots of $|\Omega(\mathbf{q}, t)|^{2}$ in the complex $t$ plane, showing the location of turning points where $\Omega(\mathbf{q}, t)=0$. These plots are for the elliptic polarization $\delta=0.5$. The other field parameters are $E_{0}=0.1 \sqrt{2} E_{c r}, \omega=0.6 m$, and $\tau=10 / m$. From top left to bottom right the values of the large chirp parameters are $b=0,0.005,0.02,0.06 \mathrm{~m}^{2}$, respectively, and the momentum values (in units of $m$ ) are $\left(q_{x}=0, q_{y}=0\right),\left(q_{x}=0, q_{y}=0.1\right),\left(q_{x}=0.4, q_{y}=0.1\right),\left(q_{x}=-0.08, q_{y}=0.06\right)$, respectively. 
single complex-conjugated pair dominate, and the spectra of the produced particles can be qualitatively explained (see Fig. 3 in Ref. [31]). The WKB result for the created number of pairs of momentum $\mathbf{q}$ is hereby given by

$$
N_{\mathbf{q}} \approx \exp (-2 K), \quad K=\left|\int_{t_{1}}^{t_{2}} \Omega(\mathbf{q}, t) d t\right|
$$

where $t_{1}$ and $t_{2}$ are dominant turning points closed to the real $t$ axis.

In the case of $A(t) \propto 1 /\left(\omega\left(1+\omega^{2} t^{2}\right)\right)$, one obtains two pairs of complex turning points which then explain interference effects [32]. The production rate was then estimated to be a sum of two terms which takes the form

$$
N_{\mathbf{q}} \approx e^{-2 K_{1}}+e^{-2 K_{2}} \pm 2 \cos (2 \alpha) e^{-K_{1}-K_{2}}
$$
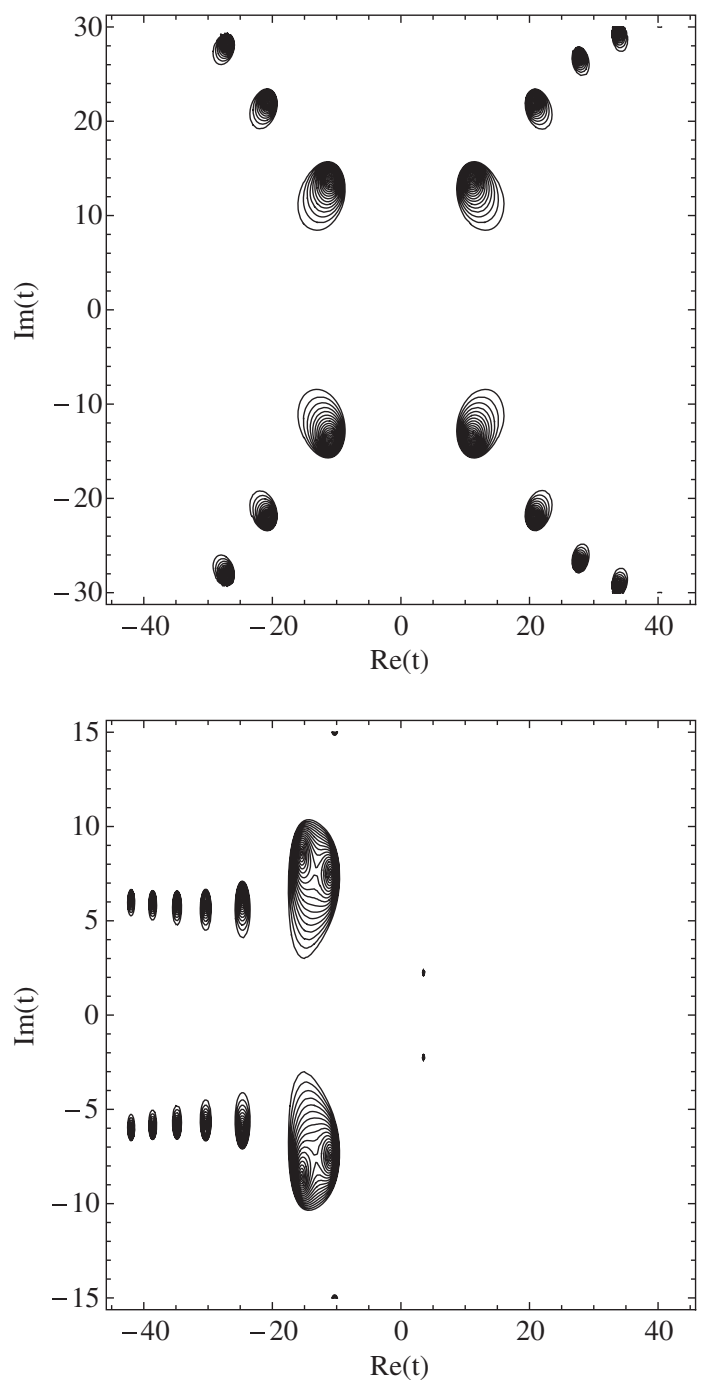

where the + and - signs refer to bosonic and fermionic pair production, respectively. Hereby, the $K_{1,2}$ are obvious generalizations of the $K$ above, and $\alpha$ represents the phase accumulated between different pairs of turning points; for details, see Ref. [32]. The interference term in Eq. (20) is responsible for the oscillations in the spectra. As the integrals $K$ and $K_{1,2}$, respectively, are almost linear in $t$, the dominant contribution originates from the terms involving turning points which are closest to the real $t$ axis.

For the gauge potential of the electric field given in Eq. (1), there exists an infinite number of complex turning points which can be obtained only numerically. In addition, as for nonvanishing chirp parameters, the momentum spectra are due to the strong interference effects peaked at quite different momenta. We follow here Ref. [29] and select for each parameter set representative values for the momenta to evaluate the turning points.
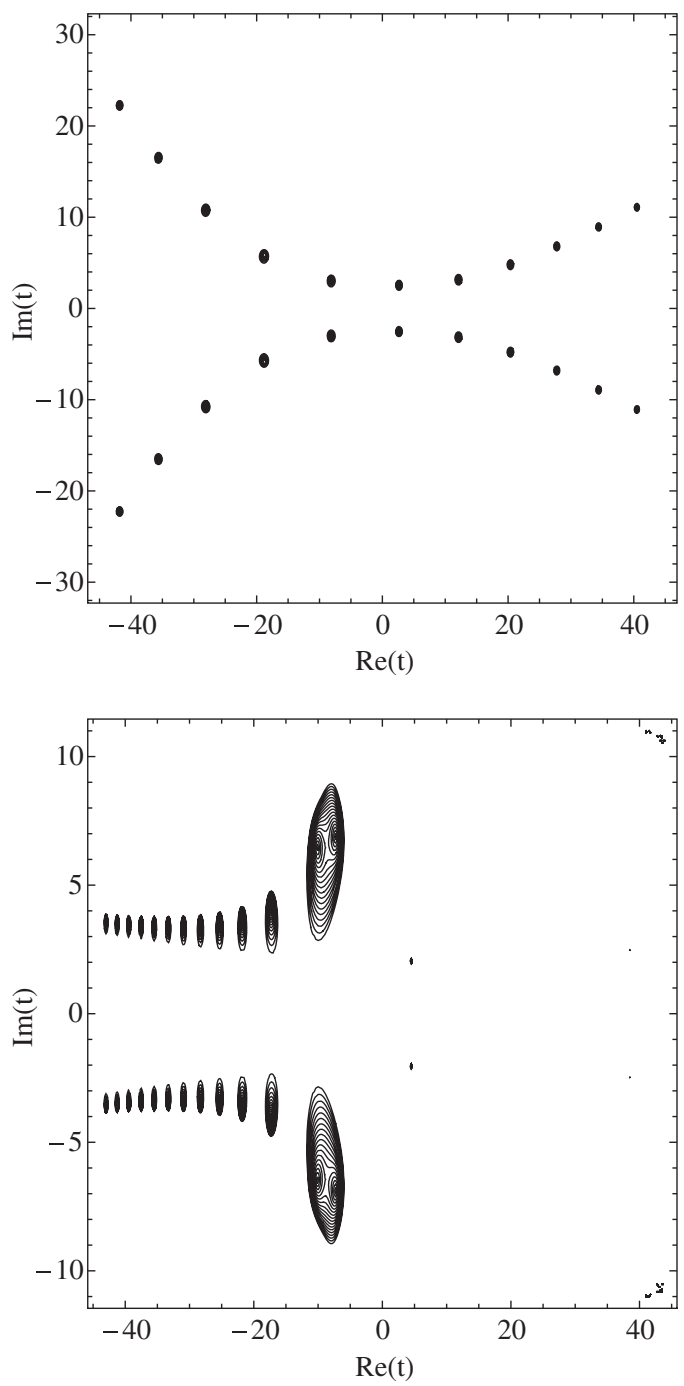

FIG. 12. Contour plots of $|\Omega(\mathbf{q}, t)|^{2}$ in the complex $t$ plane, showing the location of turning points where $\Omega(\mathbf{q}, t)=0$. These plots are for the circular polarization $\delta=1$. The other field parameters are $E_{0}=0.1 \sqrt{2} E_{c r}, \omega=0.6 m$, and $\tau=10 / m$. From top left to bottom right the values of the large chirp parameters are $b=0,0.005,0.03,0.05 \mathrm{~m}^{2}$, respectively, and momentum values (in units of $m$ ) are $\left(q_{x}=0, q_{y}=0\right),\left(q_{x}=-0.5, q_{y}=0\right),\left(q_{x}=-0.1, q_{y}=0.1\right),\left(q_{x}=0.1, q_{y}=0.01\right)$, respectively. 
For the case of linear polarization, these turning points are shown in Fig. 9. For $b=0$, there is an infinite tower of turning point pairs, but only the closest ones to the real axis contribute effectively. (From Fig. 5(a) one can infer the weak interference patterns which relate to the exponentially suppressed contributions of the other turning points.) As the chirp parameters change, the corresponding dominating pairs of turning points close to the real axis are for small values of $b$ altered mildly but for larger values very strongly; see Fig. 9. Hereby, several pairs of turning points possess a similar distance to the real axis, and therefore the appearance of strong interference effects is understood. From top panels of the Fig. 10 one can see that for $b=$ $0.03 \mathrm{~m}^{2}$ more turning point pairs appear in the complex $t$ plane for $q_{x}=-0.24 \mathrm{~m}$ as compared to $q_{x}=0.32 \mathrm{~m}$. On the other hand, in the lower panels of Fig. 10 one can infer that for $b=0.04 \mathrm{~m}^{2}$ more pairs of turning points exist for $q_{x}=$ $0.13 m$ then for $q_{x}=-0.4 m$. This provides a potential explanation why more of the produced particles possess negative (positive) momentum in $q_{x}$-direction for $b=$ $0.03 m^{2}\left(b=0.04 m^{2}\right)$, see Fig. 4. For elliptic polarization practically the same overall picture applies, see Fig. 11 with, however, some differences in the turning point positions, especially for large values of $b$.

For circular polarization the turning points are depicted in Fig. 12 for several values of chirp parameters. As we can see, for $b=0$, one of the important difference from the cases of linear polarization and elliptic polarization shown in Fig. 9(a) is that now the dominant contribution comes from the two central turning points because they are equally distant from the real axis. The two turning point pairs explain the (weak) interference effect observed for circular polarization and $b=0$. Increasing now, the chirp parameter leads to quite a distinctive pattern of turning points, as in the other two polarizations, and explains thus the differences in response to the chirp parameter for the circular versus linear or more general polarization.

\section{SUMMARY AND CONCLUSIONS}

Within the real-time DHW formalism, we studied the effect of linear frequency chirps for four polarizations, namely linear, elliptic, near-circular elliptic and circular polarization. The main results for the number densities and spectra of produced pairs can be summarized as follows.

For an electric field with a linear polarization field, the produced pairs' spectra exhibit a shift and split of peaks as well as strong interference effects as the chirp parameter increases. The most complex pattern for increasing chirps occurs, not unexpectedly, for an elliptic polarization. For the near-circular elliptic and the circular polarization, the ring form of the spectrum present at vanishing chirps is distorted, spiral structures appear and eventually, for very large chirp parameters, the peak is shifted to the central region.

The most important finding, however, is the very strong increase in number densities when the chirp parameter is increased. For vanishing and small chirps, we have verified the known differences in number densities for different polarizations, with the largest number density achieved by a linearly polarized field. Also, quite unexpectedly, this effect goes away for larger chirps, and the number densities for different polarizations become degenerate.

In this exploratory study, we restricted ourselves to a quite large value of the electric field and a quite short pulse duration. To verify or falsify a possible interpretation of the increase in the number of produced pairs in terms of an onset of multiphoton pair production, a study with much longer pulses would be necessary. Given the steep increase in the produced number pair densities and the related improved potential for an experimental observation, the effort of a study employing smaller values of the electric field and much longer pulse times is certainly justified.

\section{ACKNOWLEDGMENTS}

We thank Christian Kohlfürst for a critical reading of the manuscript and helpful remarks. This work was supported by the National Natural Science Foundation of China (NSFC) under Grants No. 11875007, No. 11705278 and No. 11475026. The computation was carried out at the HSCC of the Beijing Normal University.
[1] F. Sauter, Z. Phys. 69, 742 (1931).

[2] W. Heisenberg and H. Euler, Z. Phys. 98, 714 (1936).

[3] J. S. Schwinger, Phys. Rev. 82, 664 (1951).

[4] F. Gelis and N. Tanji, Prog. Part. Nucl. Phys. 87, 1 (2016).

[5] T. Heinzl and A. Ilderton, Eur. Phys. J. D 55, 359 (2009).

[6] M. Marklund and J. Lundin, Eur. Phys. J. D 55, 319 (2009).

[7] O. J. Pike, F. Mackenroth, E. G. Hill, and S. J. Rose, Nat. Photonics 8, 434 (2014).
[8] A. Ringwald, Phys. Lett. B 510, 107 (2001).

[9] R. Alkofer, M. B. Hecht, C. D. Roberts, S. M. Schmidt, and D. V. Vinnik, Phys. Rev. Lett. 87, 193902 (2001).

[10] C. D. Roberts, S. M. Schmidt, and D. V. Vinnik, Phys. Rev. Lett. 89, 153901 (2002).

[11] R. Schützhold, H. Gies, and G. Dunne, Phys. Rev. Lett. 101, 130404 (2008); M. Orthaber, F. Hebenstreit, and R. Alkofer, Phys. Lett. B 698, 80 (2011); B. King, H. Gies, and 
A. Di Piazza, Phys. Rev. D 86, 125007 (2012); T. Nousch, D. Seipt, B. Kampfer, and A. I. Titov, Phys. Lett. B 715, 246 (2012); I. Akal, S. Villalba-Chávez, and C. Müller, Phys. Rev. D 90, 113004 (2014); C. Schneider and R. Schützhold, Phys. Rev. D 94, 085015 (2016); G. Torgrimsson, J. Oertel, and R. Schützhold, Phys. Rev. D 94, 065035 (2016).

[12] F. Hebenstreit, R. Alkofer, G. V. Dunne, and H. Gies, Phys. Rev. Lett. 102, 150404 (2009); F. Hebenstreit, R. Alkofer, and H. Gies, Phys. Rev. D 78, 061701 (2008).

[13] N. Abdukerim, Z. L. Li, and B. S. Xie, Phys. Lett. B 726, 820 (2013).

[14] D. Vasak, M. Gyulassy, and H. T. Elze, Ann. Phys. (N.Y.) 173, 462 (1987); I. Bialynicki-Birula, P. Górnicki, and J. Rafelski, Phys. Rev. D 44, 1825 (1991); F. Hebenstreit, R. Alkofer, and H. Gies, Phys. Rev. D 82, 105026 (2010); F. Hebenstreit, R. Alkofer, and H. Gies, Phys. Rev. Lett. 107, 180403 (2011).

[15] F. Hebenstreit, Ph.D. thesis, arXiv:1106.5965.

[16] A. Blinne and H. Gies, Phys. Rev. D 89, 085001 (2014); A. Blinne and E. Strobel, Phys. Rev. D 93, 025014 (2016).

[17] A. Blinne, Ph.D. thesis, arXiv:1701.00743.

[18] Z. L. Li, D. Lu, and B. S. Xie, Phys. Rev. D 92, 085001 (2015).

[19] A. N. Pfeiffer, C. Cirelli, M. Smolarski, D. Dimitrovski, M. Abu-samha, L. B. Madsen, and U. Keller, Nat. Phys. 8, 76 (2012).
[20] D. Strickland and G. Mourou, Opt. Commun. 56, 219 (1985).

[21] L. V. Keldysh, Sov. Phys. JETP 20, 1307 (1965).

[22] C. Kohlfürst, Ph.D. thesis, arXiv:1512.06082.

[23] C. Kohlfürst and R. Alkofer, Phys. Lett. B 756, 371 (2016).

[24] D. Berényi and P. Lévai, Phys. Lett. B 782, 162 (2018).

[25] Z. L. Li, Y. J. Li, and B. S. Xie, Phys. Rev. D 96, 076010 (2017).

[26] K. Krajewska and J. Z. Kamiński, Phys. Rev. A 86, 052104 (2012); S. Augustin and C. Müller, J. Phys. Conf. Ser. 497, 012020 (2014).

[27] A. Wöllert, H. Bauke, and C. H. Keitel, Phys. Rev. D 91, 125026 (2015); F. Fillion-Gourdeau, F. Hebenstreit, D. Gagnon, and S. MacLean, Phys. Rev. D 96, 016012 (2017).

[28] A. N. Pfeiffer, C. Cirelli, A. S. Landsman, M. Smolarski, D. Dimitrovski, L. B. Madsen, and U. Keller, Phys. Rev. Lett. 109, 083002 (2012).

[29] C. K. Dumlu, Phys. Rev. D 82, 045007 (2010).

[30] C. Kohlfürst, H. Gies, and R. Alkofer, Phys. Rev. Lett. 112, 050402 (2014).

[31] E. Akkermans and G. V. Dunne, Phys. Rev. Lett. 108, 030401 (2012).

[32] C. K. Dumlu and G. V. Dunne, Phys. Rev. Lett. 104, 250402 (2010). 